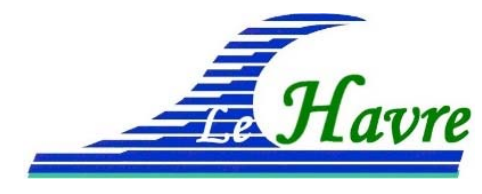

XVI ìmes Journées Nationales Génie Côtier - Génie Civil

Le Havre, 2020

DOI:10.5150/jngcgc.2020.008 C Editions Paralia CFL

disponible en ligne - http://www.paralia.fr - available online

\title{
Modélisation hydrodynamique des nappes d'hydrocarbures en zones côtières
}

\author{
Nisrine IOUZZI ${ }^{1}$, Mehdi HAFFANE ${ }^{1}$, Mohamed CHAGDALI ${ }^{1}$
}

\author{
1. LPPPC, Faculté des Sciences Ben M'Sik, Université Hassan II Casablanca \\ iouzzi.nisrine@gmail.com ; mehdihaffane@hotmail.fr ; \\ chagdalimohamed@gmail.com
}

\section{Résumé :}

La pollution marine accidentelle par les hydrocarbures est l'une des questions importantes qui préoccupent les états côtiers, vu la complexité et la difficulté de sa gestion en cas de son occurrence, en sus des conséquences écologiques et socio-économiques qui peuvent s'avérer dramatiques.

Le présent travail porte sur la modélisation numérique de la dérive et de l'étalement des nappes d'hydrocarbures sous l'action de la circulation marine et des conditions météorologiques. Le modèle choisi est le système TELEMAC. Le site d'étude est l'embouchure d'Azemmour qui se trouve sur la façade atlantique du Maroc.

Les simulations réalisées ont permis d'identifier, entre autres, le temps du déplacement de la nappe et les zones côtières qui seront particulièrement touchées par la pollution dite "marée noire". La simulation ainsi réalisée est un outil de prédiction et d'observation important du comportement des nappes d'hydrocarbures de la zone côtière, pour la mise en place des plans de gestion et de prévention.

\section{Mots clés :}

Pollution marine accidentelle, Nappe d'hydrocarbures, Modèles numériques, TELEMAC, Plan de gestion et de prévention.

\section{Introduction}

La majeure partie du pétrole transporté (62\%) utilise le transport maritime, qui suit des itinéraires très spécifiques et en empruntant des passages stratégiques qui sont communément appelés "choke point". Le transport maritime des hydrocarbures au Maroc, a connu une évolution positive pendant les vingt dernières années, avec une moyenne de 14 Millions de tonnes et un pic dépassant 16 Mt enregistré en 2013 et 2017.

$\mathrm{Vu}$ sa position géographique à l'intersection des lignes maritimes mondiales du transport des pétroliers, plusieurs sites au Maroc, selon l'index de sensibilité environnementale ESI (Environmental Sensitivity Index) ont été classés étant des sites à haut risque signifiant une forte probabilité d'incidents avec des conséquences importantes sur l'environnement et les activités liées au littoral (figure 1). 


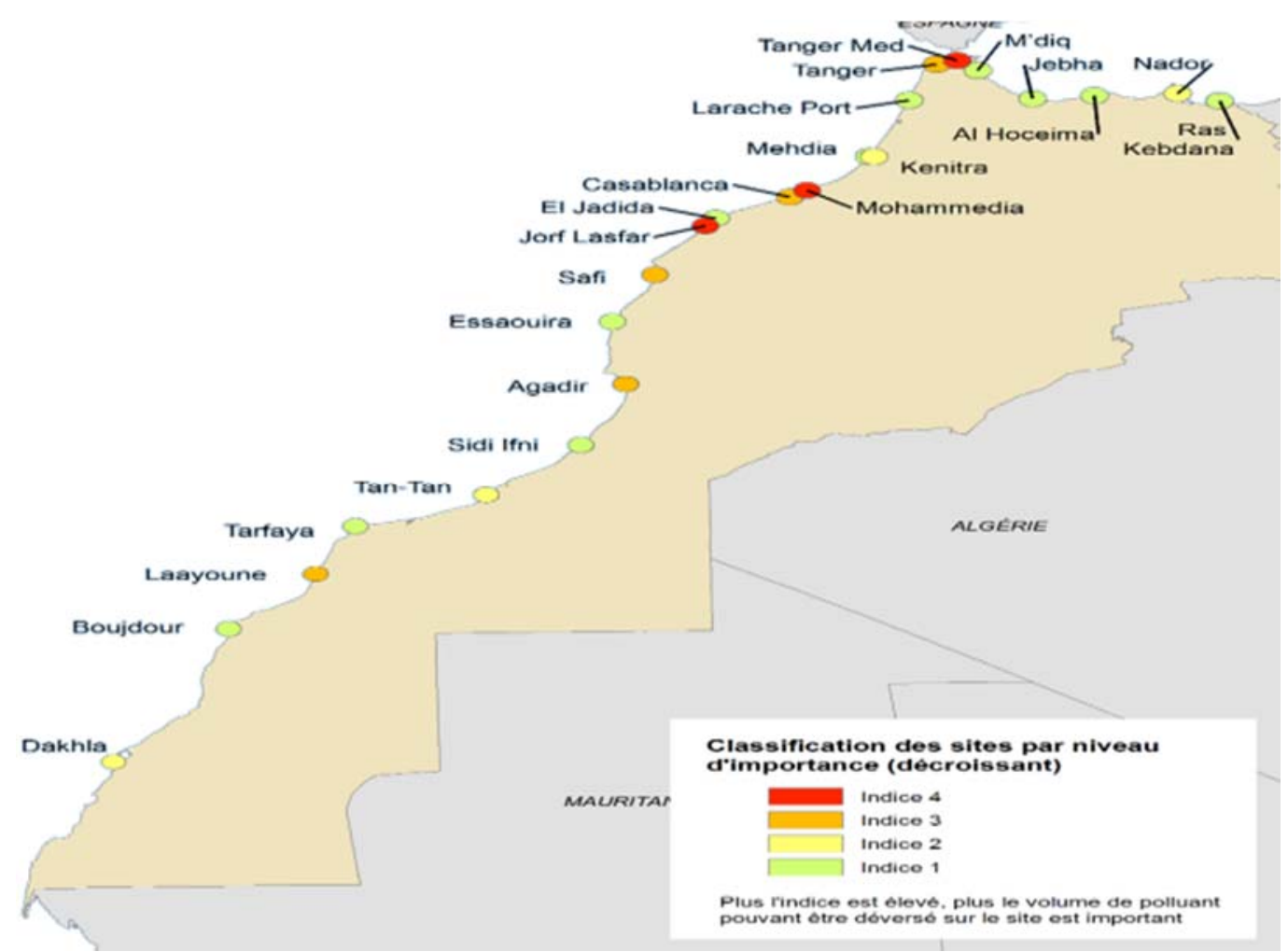

Figure 1. Classification des sites marocains à haut risque de pollution marine accidentelle, (source : Département du développement durable du Maroc).

\section{Modélisation de la dérive des nappes d'hydrocarbures}

La prédiction de dérive de nappes d'hydrocarbures nécessite le couplage des équations de transport et des cinétiques chimiques avec un modèle hydrodynamique. Le système TELEMAC est un outil de modélisation dédié aux écoulements à surface libre dans les domaines maritimes et fluviaux.

Pour notre travail, on a utilisé TELEMAC-2D (HERVOUET, 2007). Ce logiciel résout les équations de Barré de Saint-Venant à deux dimensions d'espace horizontales. Ses résultats principaux sont, en chaque point du maillage de résolution, la hauteur d'eau et la vitesse moyenne sur la verticale.

Le code TELEMAC-2D résout simultanément les quatre équations hydrodynamiques suivantes :

$$
\begin{aligned}
& \frac{\partial h}{\partial t}+\vec{u} \cdot \vec{\nabla}(h)+h \operatorname{div}(\vec{u})=S_{h} \\
& \frac{\partial h}{\partial t}+\vec{u} \cdot \vec{\nabla}(u)=-g \frac{\partial Z}{\partial X}+S_{X}+\frac{1}{h} \operatorname{div}\left(h v_{t} \vec{\nabla} u\right) \\
& \frac{\partial v}{\partial t}+\vec{u} \cdot \vec{\nabla}(v)=-g \frac{\partial Z}{\partial y}+S_{y}+\frac{1}{h} \operatorname{div}\left(\mathrm{h} v_{t} \vec{\nabla} u\right) \\
& \frac{\partial T}{\partial t}+\vec{u} \cdot \vec{\nabla}(T)=S_{T}+\frac{1}{h} \operatorname{div}\left(\mathrm{h} v_{T} \vec{\nabla} T\right)
\end{aligned}
$$




\section{XVIèmes Journées Nationales Génie Côtier - Génie Civil \\ Le Havre 2020}

avec : h $(\mathrm{m})$, hauteur d'eau; $\mathrm{u}, \mathrm{v}(\mathrm{m} / \mathrm{s})$, composantes de la vitesse $; \mathrm{T}\left(\mathrm{g} / \mathrm{l}\right.$ ou $\left.{ }^{\circ} \mathrm{C}\right)$, traceur passif; $\mathrm{g}\left(\mathrm{m} / \mathrm{s}^{2}\right)$, accélération de la pesanteur $; v_{t}, v_{T}\left(\mathrm{~m}^{2} / \mathrm{s}\right)$, coefficients de diffusion de la vitesse et du traceur; $\mathrm{Z}(\mathrm{m})$, cote de la surface libre; $\mathrm{t}(\mathrm{s})$, temps ; $\mathrm{x}, \mathrm{y}(\mathrm{m})$, composantes d'espace horizontales; $\mathrm{S}_{\mathrm{h}}(\mathrm{m} / \mathrm{s})$, source ou puits de fluide; $\mathrm{S}_{\mathrm{x}}, \mathrm{S}_{\mathrm{y}}\left(\mathrm{m} / \mathrm{s}^{2}\right)$, termes sources ou puits des équations dynamiques; $\mathrm{S}_{\mathrm{T}}(\mathrm{g} / \mathrm{l} / \mathrm{s})$, source et puits de traceur ; h, $\mathrm{u}, \mathrm{v}$ et $\mathrm{T}$ sont les inconnues.

La prévision de la dérive de la nappe d'hydrocarbures nécessite le couplage des équations de transport et des cinétiques chimiques propres à l'hydrocarbure avec le modèle hydrodynamique. La qualité des prévisions est directement reliée à la qualité des données hydrodynamiques. Les différents processus mis en jeu dans le modèle sont dits « processus de vieillissement de la nappe ». Lorsque l'hydrocarbure est déversé dans le milieu aquatique, un ensemble de processus de transport (convection et diffusion) et de transformation se déclenche.

Il est également important de prendre en compte les considérations suivantes :

- l'extrême diversité des polluants existants, pétroles bruts ou produits raffinés, fluides à pâteux, de densité très inférieure à proche de celle de l'eau de mer.

- les conditions climatiques (climat, météo).

L'évolution du polluant dépend des mécanismes qui altèrent sa composition et ses propriétés physico-chimiques : évaporation, dissolution, photo-oxydation, biodégradation, émulsification, voir figure 2 .

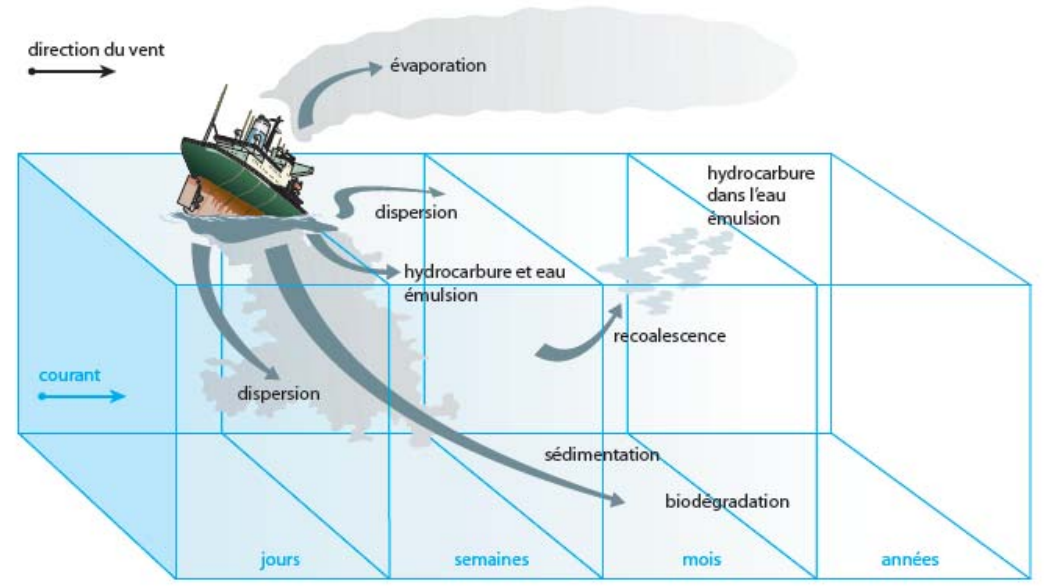

Figure 2. Processus de vieillissement de la nappe d'hydrocarbure (IOGP, 2015).

\section{Description du site du projet}

L'embouchure de l'Oued Oum Rbia se situe au niveau de la côte atlantique dans la commune d'Azemmour relevant de la province d'El Jadida au nord de Jorf Lasfar bordée du nord au sud par un cordon dunaire rectiligne ainsi qu'une plage au sud la plage d'Al Haouzia, voir figure 3. 


\section{Thème 1 - Hydrodynamique côtière}

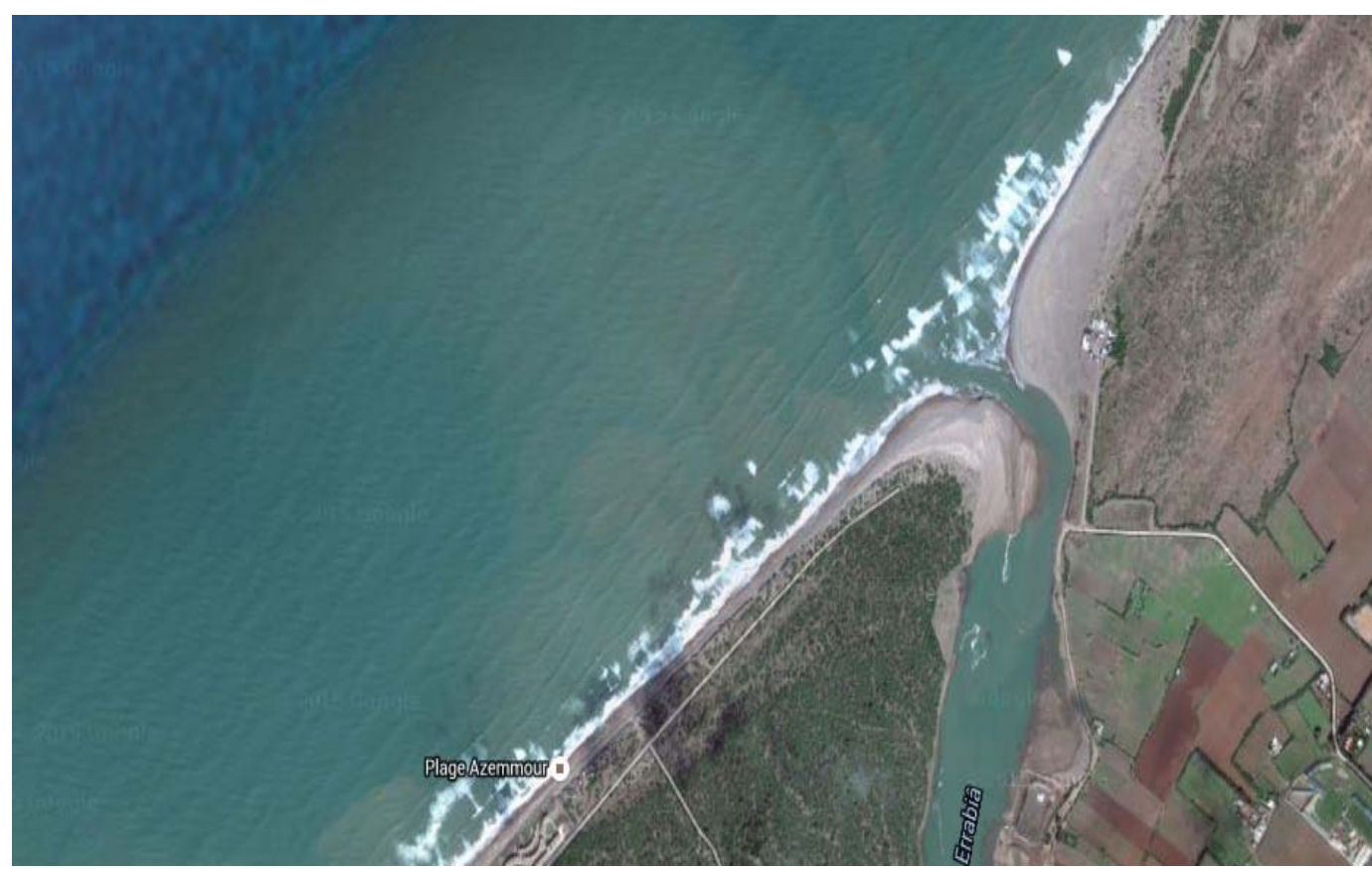

Figure 3. Site d'étude (source : Google).

La carte marine des services SHOM n6621 a été utilisée pour définir l'emprise du domaine global (figure 4). Ce domaine est considéré à -100 mètres de profondeur, une longueur de $40 \mathrm{~km}$ et la largeur est de $34 \mathrm{~km}$. L'exploitation du modèle est faite avec les sources de données pour lesquelles les positions sont généralement exprimées en Lambert. A l'intérieur du domaine global on a défini un sous domaine (voir figure 5), où nous possédons une bathymétrie plus raffinée.

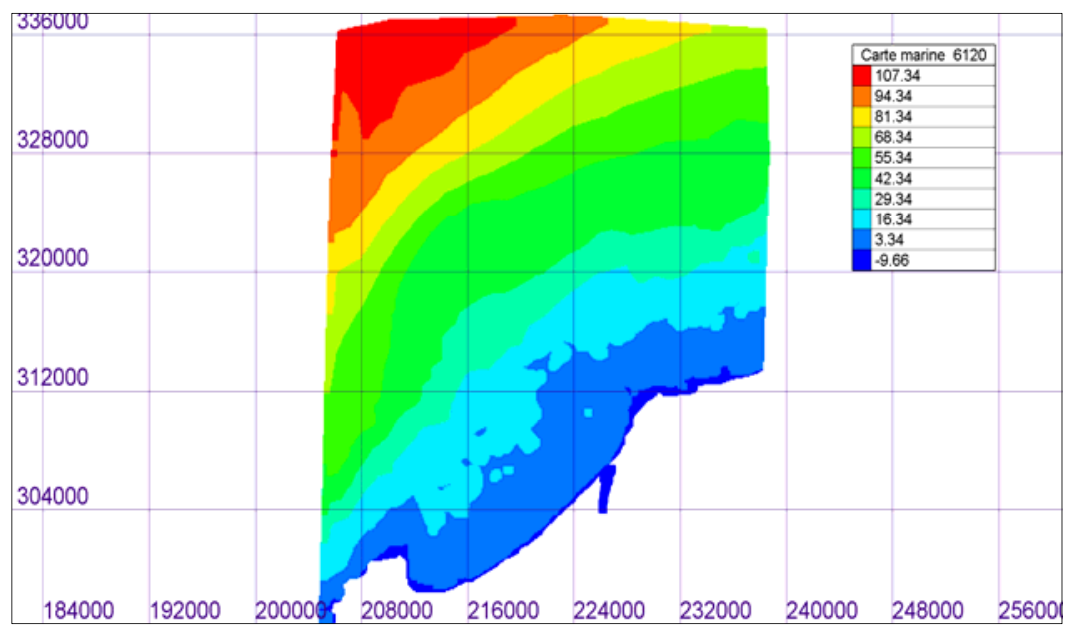

Figure 4. Carte Marine 6621-SHOM.

Le maillage est construit à partir des éléments Triangulaire à 3 nœuds (T3). Le maillage est construit en Lambert zone 93-Maroc et les niveaux d'eau sont référencés en NGM- 


\section{XVIèmes Journées Nationales Génie Côtier - Génie Civil \\ Le Havre 2020}

Maroc. On évite ainsi de devoir utiliser un repère local, ce qui facilite l'exploitation du modèle avec les sources de données pour lesquelles les positions sont généralement exprimées en Lambert zone 4. Les conditions aux limites utilisées dans notre modèle sont les suivantes : (i) frontière liquide à débit imposé correspondant au débit de crue de l'estuaire dans la limite inférieure, (ii) frontière liquide à hauteur imposée dans la limite supérieure et des frontières liquides à hauteur libre dans les deux autres côtés. On considère que le vent est constant dans l'espace et dans le temps. Les valeurs algébriques des vitesses du vent suivant l'axe $\mathrm{X}$ et l'axe $\mathrm{Y}$ à prendre dans le modèle sont respectivement de $-1 \mathrm{~m} / \mathrm{s}$ et $1.3 \mathrm{~m} / \mathrm{s}$.

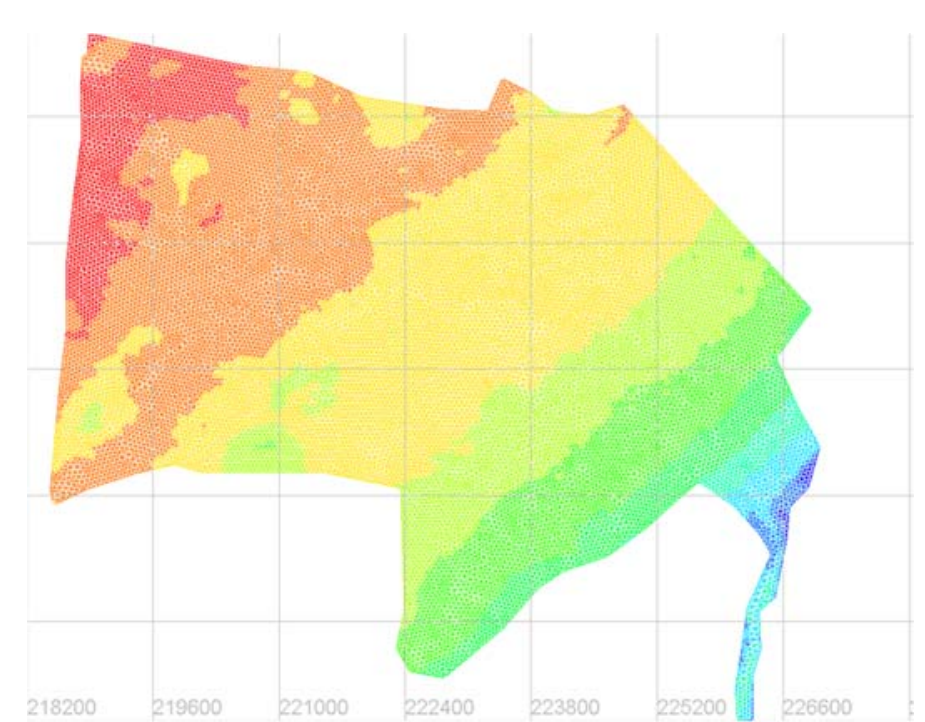

Figure 5. Emprise du domaine d'étude avec le maillage.

\section{Résultats de la simulation}

Après la validation du modèle sur des configurations tests (voir manuel utilisateur, version 7p2, TELEMAC), le modèle a été appliqué sur le modèle définissant le site d'étude (figure 5). Les résultats des deux composantes de la vitesse sont illustrés sur les figures 6 et 7. Les hauteurs d'eau sont présentées sur la figure 8 . 


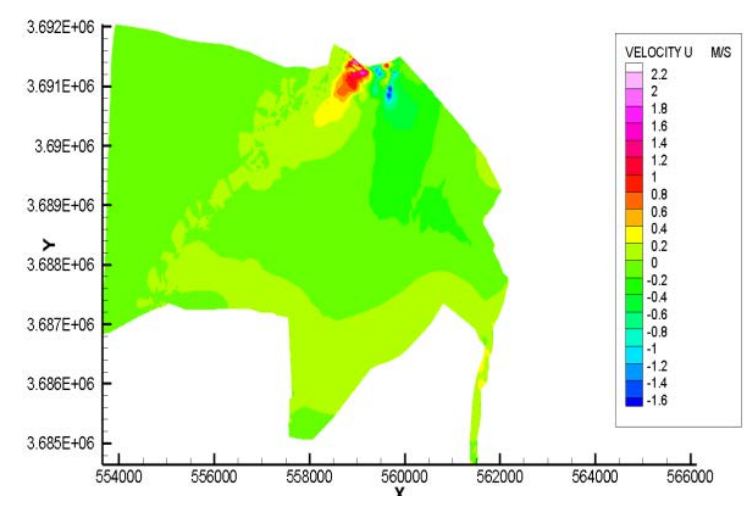

Figure 6. Profil de la vitesse selon $x$.

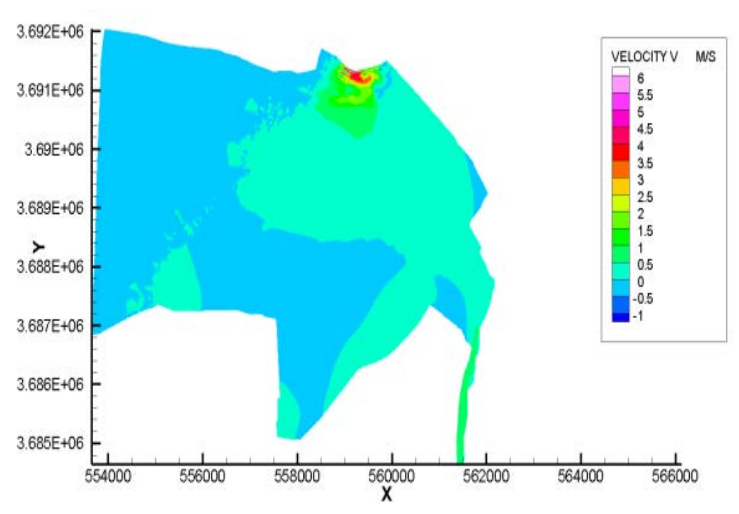

Figure 7. Profil de la vitesse selon y.

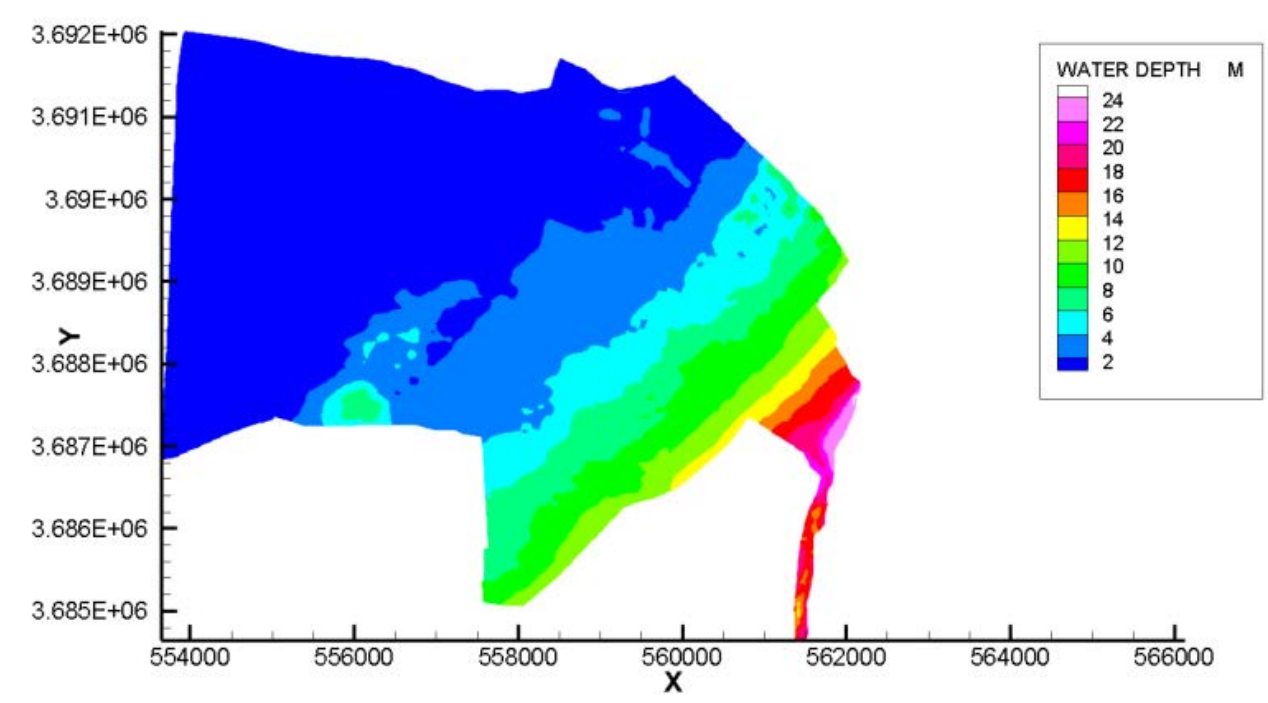

Figure 8. Profil de la profondeur d'eau.

En introduisant le point de déversement par les hydrocarbures à l'embouchure de l'Oum Rbia dans notre zone d'étude par utilisation du modèle MIGR'HYCAR intégré à TELEMAC. La simulation hydrodynamique en présence du vent, démontre son fractionnement avec le déplacement d'une partie vers la côte et, ce dès le deuxième jour de simulation (figure 9). La simulation de la dissolution du premier composant soluble de la nappe d'hydrocarbure démontre les fortes concentrations au niveau de la côte en deux zones différentes (figures 10 et 11). 


\section{XVİ̀mes Journées Nationales Génie Côtier - Génie Civil \\ Le Havre 2020}

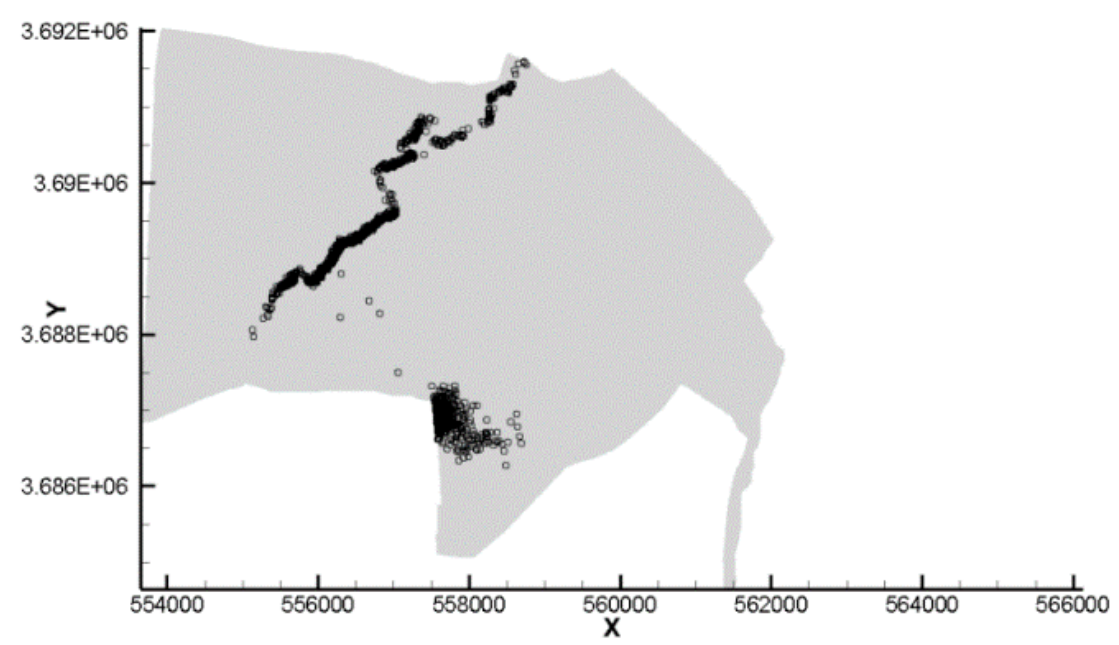

Figure 9. Dérive de la nappe d'hydrocarbures en présence du vent.

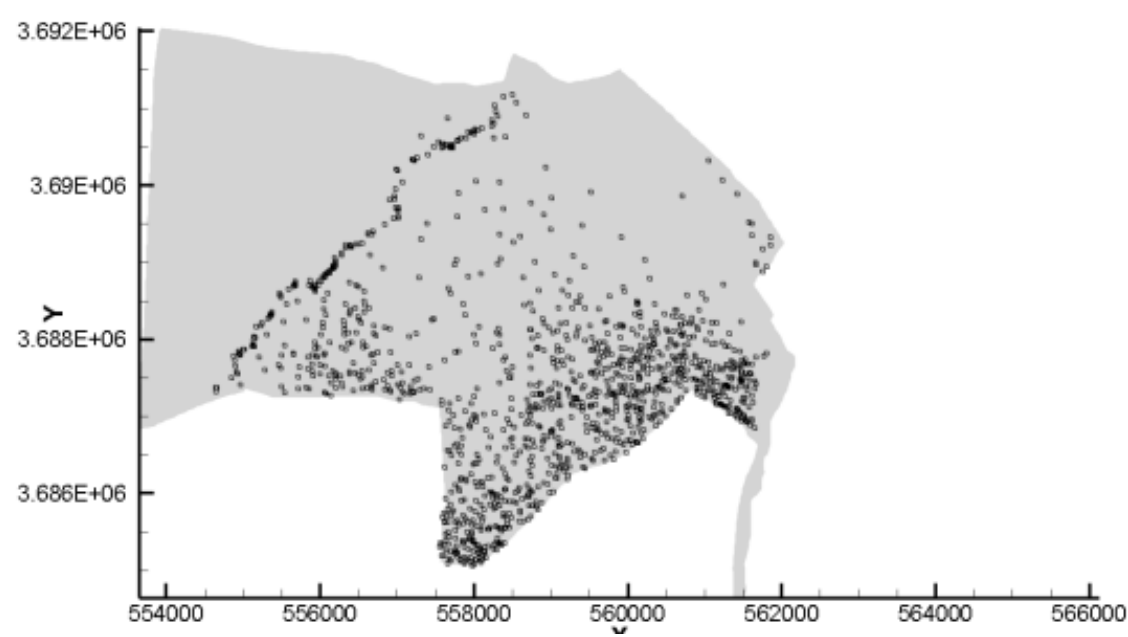

Figure 10. Dérive de la nappe d'hydrocarbures sans l'effet du vent.

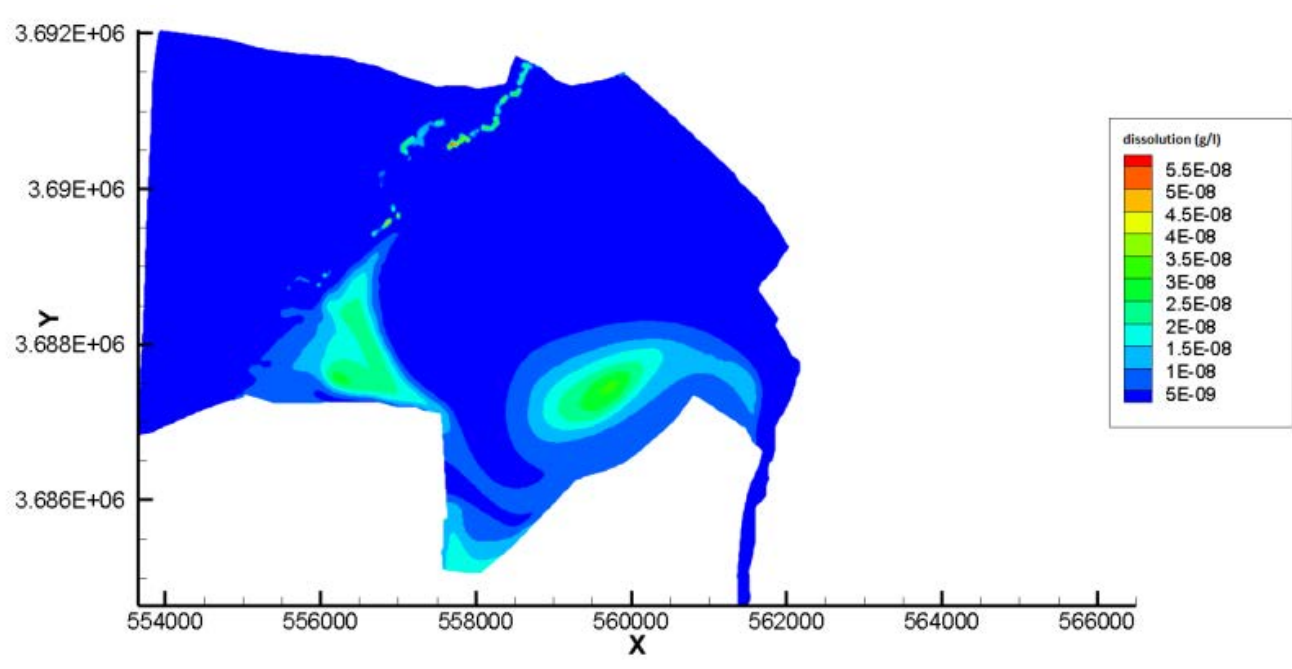

Figure 11. Dissolution du premier traceur. 


\section{Thème 1 - Hydrodynamique côtière}

\section{Conclusion}

La prise en conscience de la pollution, en particulier la pollution marine accidentelle par les hydrocarbures, de la part de l'opinion publique et des pouvoirs en place a fait de ce phénomène un problème d'actualité méritant une grande réflexion. Comme c'est impossible d'arrêter cette pollution, il faut pouvoir lutter contre ses conséquences négatives. La connaissance à l'avance du comportement d'un produit déversé accidentellement est un élément indispensable pour définir les options de lutte et appréhender la nature de l'impact environnemental. Ce travail a permis de dresser l'état de l'art en la matière, et de développer un modèle intégré dans TELEMAC avec une application dans la région d'Azemmour qui a permis de simuler l'évolution de l'étalement et la trajectoire de la nappe d'hydrocarbure et disposer ainsi d'un outil de gestion et de prévention très pertinent pour une bonne gouvernance des zones maritimes et côtières.

\section{Références bibliographiques}

HERVOUET J.-M. (2007). Hydrodynamics of free surface flows. Modelling with the finite element method, Wiley, $360 \mathrm{p}$.

IOGP -Association internationale des producteurs d'hydrocarbures et de gaz- (2015). Observation aérienne des déversements d'hydrocarbures en mer. Guide de bonnes pratiques en matière de gestion des incidents et de personnel d'intervention d'urgence. Rapport de l'IOGP, numéro 518, 56 p. 